Georgian Mathematical Journal

Volume 13 (2006), Number 2, 251-260

\title{
THE POTENTIAL METHOD FOR THE REACTANCE WAVE DIFFRACTION PROBLEM IN A SCALE OF SPACES
}

\author{
LUIS P. CASTRO AND DAVID NATROSHVILI
}

\begin{abstract}
This paper is concerned with a screen type boundary value problem arising from the wave diffraction problem with a reactance condition. We consider the problem in a weak formulation within Bessel potential spaces, and where both cases of a complex and a pure real wave number are analyzed. Using the potential method, the boundary value problem is converted into a system of integral equations. The invertibility of the corresponding matrix pseudodifferential operator is shown in appropriate function spaces which allows the conclusion about the existence and uniqueness of a weak solution to the original problem. Higher regularity properties of solutions are also proved to exist in some scale of Bessel potential spaces, upon the corresponding smoothness improvement of given data. In particular, the $C^{\alpha}$-smoothness of solutions in a neighbourhood of the screen edge is established with arbitrary $\alpha<1$ in the two-dimensional case and $\alpha<1 / 2$ in the three-dimensional case.
\end{abstract}

2000 Mathematics Subject Classification: 35J05, 35J25, 35P25.

Key words and phrases: Helmholtz equation, wave diffraction, reactance condition, integral equations, potential theory.

\section{Introduction and the Formulation of the Problem}

We consider a boundary-transmission problem for the Helmholtz equation, in the Bessel potential space setting, which arises in the context of wave diffraction theory. The boundary $\Sigma$ under consideration consists of a (possibly distorted) strip (along the $x_{3}$-axis) and certain boundary conditions are assumed on it in the form of so-called reactance conditions $[1,2,3]$.

For a large range of materials the electromagnetic theory gives a quasihomogeneous refracted wave, which propagates perpendicularly to the boundary $\Sigma$ irrespective of an incident angle. The $x_{3}$-dependence can be therefore cancelled due to the perpendicular wave propagation implying a two-dimensional formulation of the diffraction problem in question.

Let $\Omega:=\mathbb{R}^{2} \backslash \bar{\Sigma}$, where $\Sigma$ is a $C^{\infty}$-smooth non-self-intersecting connected open arc (say, a straight line segment $(0, a)$ on the $x$-axis). We assume that $\Sigma$ is a proper part of some closed $C^{\infty}$-smooth simple curve $S$ surrounding some bounded domain $\Omega^{+}$. Let $\Omega^{-}:=\mathbb{R}^{2} \backslash \overline{\Omega^{+}}$. By $n(x)=\left(n_{1}(x), n_{2}(x)\right)$ we denote the outward unit normal vector at a point $x \in S=\partial \Omega^{+}$. This defines the positive direction of the normal vector on the arc $\Sigma$ uniquely.

We look for a weak solution $u \in H_{l o c}^{1}(\Omega)$ to the Helmholtz equation

$$
\left(\Delta+k^{2}\right) u=0 \text { in } \Omega \text {, }
$$

ISSN 1072-947X / \$8.00 / C Heldermann Verlag www.heldermann.de 
satisfying the following boundary conditions on $\Sigma$

$$
\begin{aligned}
& {[u(x)]^{+}-[u(x)]^{-}=h_{0}(x), \quad x \in \Sigma,} \\
& {\left[\partial_{n} u(x)\right]^{+}-\left[\partial_{n} u(x)\right]^{-}+q[u(x)]^{+}=h_{1}(x), \quad x \in \Sigma,}
\end{aligned}
$$

where $\partial_{n}$ denotes the normal derivative operator, and the wave number $k$ and the reactance number $q$ are in general complex constants $k=k_{1}+i k_{2}, q=$ $q_{1}+i q_{2}$ (with $k_{j}, q_{j} \in \mathbb{R}$ ); we assume that the boundary data belong to the appropriate function spaces:

$$
h_{0} \in r_{\Sigma} \widetilde{H}^{\frac{1}{2}}(\Sigma), \quad h_{1} \in r_{\Sigma} \widetilde{H}^{-\frac{1}{2}}(\Sigma),
$$

where $r_{\Sigma}$ is the restriction operator to $\Sigma$. The motivation for the inclusions (1.4) is quite natural and described in Section 3. The symbols $[\cdot]^{ \pm}$denote limits on $\Sigma$ from $\Omega^{ \pm}$which are understood either in the usual trace sense for Dirichlet type conditions or in the generalized trace sense for Neumann type conditions (see, e.g., $[4,5,6]$ ).

Here and in what follows we use the abbreviations: $H_{2, l o c}^{1}(\Omega)=H_{l o c}^{1}(\Omega)$, $\widetilde{H}_{2}^{s}(\Sigma)=\widetilde{H}^{s}(\Sigma)$, and $H_{2}^{s}(\Sigma)=H^{s}(\Sigma)$. Recall that

$$
H^{s}(\Sigma):=\left\{r_{\Sigma} f: f \in H^{s}(S)\right\}, \quad \widetilde{H}^{s}(\Sigma):=\left\{f \in H^{s}(S): \operatorname{supp} f \subset \bar{\Sigma}\right\} .
$$

Note that if $k_{2}=\Im k \neq 0$ (say $k_{2}>0$ ), then polynomially bounded solutions of the Helmholtz equation decay exponentially at infinity and we have $u \in$ $H^{1}(\Omega)$.

If $k$ is real (say $k>0$ ), then we have to require that $u \in H_{l o c}^{1}(\Omega)$ satisfy the Sommerfeld radiation condition at infinity, $u \in \operatorname{Som}(\Omega)$ (see, e.g., $[7,8]$ ):

$$
\frac{\partial}{\partial|x|} u(x)-i k u(x)=\mathcal{O}\left(|x|^{-\frac{3}{2}}\right) \quad \text { as } \quad|x| \rightarrow \infty .
$$

In what follows we assume that $u$ exponentially decays at infinity for a complex $k$, while $u$ is assumed to be radiating for a real $k$.

We will refer to the above formulated boundary value problem (BVP) as the Problem $(P)_{\Sigma}$.

In the present paper, by using a different approach we present a generalization of the results of [9] on the Problem $(P)_{\Sigma}$ along the following lines: (i) our consideration here is not restricted only to the complex wave number case but also involves the case of a wave number with a zero imaginary part; (ii) as mentioned above, we consider here surfaces other than the strip geometry analyzed in [9]; (iii) a new improvement of the high regularity scale of solutions is given in the concluding section. Note that the methods of [9] lead also to existence and uniqueness results for regularity classes given below for the weak solution case.

Finally, the approach employed in the paper can be applied to the general three-dimensional case as well, where $\Sigma$ is a two-dimensional two-sided $C^{\infty}$ smooth connected manifold of arbitrary geometrical shape with $C^{\infty}$-smooth edge boundary $\partial \Sigma$. We assume that $\Sigma$ is a proper part of some closed connected non-self-intersecting $C^{\infty}$-smooth surface $S$ surrounding a bounded domain $\Omega^{+} \in$ 
$\mathbb{R}^{3}$. In the three-dimensional formulation of the $\operatorname{BVP}(P)_{\Sigma}$ we have to replace only the Sommerfeld radiation condition (1.5) by the following one $[7,8]$

$$
\frac{\partial}{\partial|x|} u(x)-i k u(x)=\mathcal{O}\left(|x|^{-2}\right) \quad \text { as } \quad|x| \rightarrow \infty .
$$

See also Remark 4.5 below concerning the three-dimensional case.

\section{UNiqueness Result}

We start by analyzing the conditions for the uniqueness of a solution. For this, we consider the corresponding homogeneous Problem $(P)_{\Sigma}$.

Theorem 2.1. Let $k_{2}=\Im k \neq 0$, and assume that one of the following five conditions is satisfied:

1) $k_{1} k_{2} q_{2}<0$

2) $q_{2}=0, k_{1} \neq 0$

3) $k_{1}=0, q_{2} \neq 0$,

4) $k_{1}=0, q_{2}=0, q_{1} \geq 0$,

5) $k_{1} k_{2} q_{2}>0$

$\left(k_{2}^{2}-k_{1}^{2}\right)+\frac{2 k_{1} k_{2} q_{1}}{q_{2}} \geq 0$

Then the homogeneous Problem $(P)_{\Sigma}\left(h_{0}=h_{1}=0\right)$ possesses only a trivial solution.

Proof. Let $R$ be a sufficiently large positive number and $B(R)$ be the ball (disk) centered at the origin and having radius $R$. We assume that $\overline{\Omega^{+}} \subset B(R)$ and set $\Omega_{R}^{-}:=\Omega^{-} \cap B(R)$. Further, let $u$ be a solution to the homogeneous problem $(P)_{\Sigma}$ and write the corresponding Green formulae for $u$ and its complex conjugate $\bar{u}$ in the domains $\Omega^{+}$and $\Omega_{R}^{-}$(see, e.g., [8]):

$$
\begin{aligned}
& \int_{\Omega^{+}}\left[|\nabla u|^{2}-k^{2}|u|^{2}\right] d x=\left\langle\left[\partial_{n} u\right]_{S}^{+},[\bar{u}]_{S}^{+}\right\rangle_{S}, \\
& \int_{\Omega_{R}^{-}}\left[|\nabla u|^{2}-k^{2}|u|^{2}\right] d x=-\left\langle\left[\partial_{n} u\right]_{S}^{-},[\bar{u}]_{S}^{-}\right\rangle_{S}+\int_{\partial B(R)} \partial_{n} u \bar{u} d S,
\end{aligned}
$$

where $\nabla=\left(\partial_{1}, \partial_{2}\right)$. Throughout the paper the symbols $\langle\cdot, \cdot\rangle_{S}$ and $\langle\cdot, \cdot\rangle_{\Sigma}$ stand for the duality brackets between the dual spaces $H^{-\frac{1}{2}}(S)$ and $H^{\frac{1}{2}}(S)$, or $\widetilde{H}^{-\frac{1}{2}}(\Sigma)$ and $H^{\frac{1}{2}}(\Sigma)$ or $H^{-\frac{1}{2}}(\Sigma)$ and $\widetilde{H}^{\frac{1}{2}}(\Sigma)$. For regular functions, e.g., $f, g \in L_{2}(\mathcal{M})$, we have

$$
\langle f, g\rangle_{\mathcal{M}}=\int_{\mathcal{M}} f g d \mathcal{M},
$$

where $\mathcal{M} \in\{S, \Sigma\}$.

Note that due to the homogeneous boundary condition (1.2) and the interior $C^{\infty}$-regularity in $\Omega$ of solutions to the equation (1.1), we actually have $[u]_{S}^{+}=$ $[u]_{S}^{-}$. Moreover, in accordance with this interior regularity we have $\left[\partial_{n} u\right]_{S \backslash \bar{\Sigma}}^{+}=$ $\left[\partial_{n} u\right]_{S \backslash \bar{\Sigma}}^{-}$and therefore

$$
\left[\partial_{n} u\right]_{\Sigma}^{+}-\left[\partial_{n} u\right]_{\Sigma}^{-} \in \widetilde{H}^{-\frac{1}{2}}(\Sigma)
$$


Using these relations and the boundary condition (1.3), from (2.2) and (2.3) by summing we get

$$
\begin{aligned}
\int_{\Omega^{+} \cup \Omega_{R}^{-}}\left[|\nabla u|^{2}-k^{2}|u|^{2}\right] d x & =\left\langle\left[\partial_{n} u\right]_{\Sigma}^{+}-\left[\partial_{n} u\right]_{\Sigma}^{-},[\bar{u}]_{\Sigma}^{+}\right\rangle_{\Sigma}+\int_{\partial B(R)} \partial_{n} u \bar{u} d S \\
& =-q \int_{\Sigma}\left|[u]^{+}\right|^{2} d S+\int_{\partial B(R)} \partial_{n} u \bar{u} d S
\end{aligned}
$$

Since $k_{2}=\Im k \neq 0$, the function $u$ exponentially decays at infinity and in (2.4) we can pass to the limit as $R \rightarrow \infty$ to obtain

$$
\int_{\Omega}\left[|\nabla u|^{2}-k^{2}|u|^{2}\right] d x=-q \int_{\Sigma}\left|[u]^{+}\right|^{2} d S .
$$

By separating the real and the imaginary part we arrive at the equalities

$$
\begin{aligned}
\int_{\Omega}\left[|\nabla u|^{2}+\left(k_{2}^{2}-k_{1}^{2}\right)|u|^{2}\right] d x & =-q_{1} \int_{\Sigma}\left|[u]^{+}\right|^{2} d S \\
2 k_{1} k_{2} \int_{\Omega}|u|^{2} d x & =q_{2} \int_{\Sigma}\left|[u]^{+}\right|^{2} d S .
\end{aligned}
$$

Now it is very easy to verify that each condition in (2.1) yields $u=0$ in $\Omega$.

Theorem 2.2. Let $k$ be a real positive number and $q_{2}=\Im q \leq 0$. Then the homogeneous Problem $(P)_{\Sigma}\left(h_{0}=h_{1}=0\right)$ possesses only a trivial solution.

Proof. By the same arguments as above we arrive at the relation (2.4) for a solution $u$ to the homogeneous problem $(P)_{\Sigma}$. Assuming $R$ to be sufficiently large we can apply the Sommerfeld radiation condition on the sphere (circle) $\partial B(R)$ and afterwards separate the imaginary part of the equation to obtain

$$
-q_{2} \int_{\Sigma}\left|[u]^{+}\right|^{2} d S+k \int_{\partial B(R)}|u|^{2} d S=\mathcal{O}\left(R^{-1}\right) .
$$

We have employed here the fact that if $u \in \operatorname{Som}(\Omega)$, then $u(x)=\mathcal{O}\left(|x|^{-1 / 2}\right)$ as $|x| \rightarrow \infty[7]$. From (2.8) we derive

$$
\lim _{R \rightarrow \infty} \int_{\partial B(R)}|u|^{2} d S=0
$$

whence $u=0$ in $\Omega$ follows due to the well known Rellich-Vekua theorem [7, 8]. 


\section{Potentials and the Integral Representation Formula of SOLUTIONS}

Let us denote by $\Gamma(x, k):=-\frac{i}{4} H_{0}^{(1)}(k|x|)$ the fundamental function of the Helmholtz operator, $\left(\Delta+k^{2}\right) \Gamma(x, k)=\delta(x)$, where $\delta(\cdot)$ is the Dirac delta function. Here $H_{0}^{(1)}(k|x|)$ is the Hankel function of first kind and of order zero. It decays exponentially at infinity for a complex $k$ and satisfies the Sommerfeld radiation condition for a real positive $k$. We recall that the fundamental function $\Gamma(x, k)$ has a logarithmic singularity in a neighbourhood of the origin, say, for $|x|<1 / 2$ (for details see, e.g., [8])

$$
\Gamma(x, k)=-\frac{1}{2 \pi} \ln \frac{1}{|x|}+\mathcal{O}\left(|x|^{2} \ln |x|\right), \quad|x|<1 / 2 .
$$

We then consider the corresponding single and double layer potentials,

$$
\begin{aligned}
& V(\psi)(x)=\int_{\Sigma} \Gamma(x-y, k) \psi(y) d S, \\
& W(\varphi)(x)=\int_{\Sigma}\left[\partial_{n(y)} \Gamma(x-y, k)\right] \varphi(y) d S,
\end{aligned}
$$

where $\psi$ and $\varphi$ are the density functions. By the standard arguments of Green identities we can derive the following integral representation of a radiating solution of the Helmholtz equation in the domains $\Omega^{+}$and $\Omega^{-}[7]$,

$$
\begin{gathered}
\int_{\partial \Omega^{+}}\left\{\left[\partial_{n(y)} \Gamma(x-y, k)\right][u(y)]^{+}-\Gamma(x-y, k)\left[\partial_{n} u(y)\right]^{+}\right\} d S \\
=\left\{\begin{array}{lll}
u(x) & \text { for } & x \in \Omega^{+}, \\
0 & \text { for } & x \in \Omega^{-},
\end{array}\right. \\
-\int_{\partial \Omega^{-}}\left\{\left[\partial_{n(y)} \Gamma(x-y, k)\right][u(y)]^{-}-\Gamma(x-y, k)\left[\partial_{n} u(y)\right]^{-}\right\} d S \\
=\left\{\begin{array}{lll}
0 & \text { for } & x \in \Omega^{+}, \\
u(x) & \text { for } & x \in \Omega^{-} .
\end{array}\right.
\end{gathered}
$$

By summing these equations and taking into account that in our case $u$ satisfies the continuity conditions on $S \backslash \bar{\Sigma}$

$$
[u(x)]^{+}=[u(x)]^{-}, \quad\left[\partial_{n} u(x)\right]^{+}=\left[\partial_{n} u(x)\right]^{-}, \quad x \in S \backslash \bar{\Sigma},
$$

we obtain the general integral representation for a radiating solution $u$ of the equation (1.1) in the domain $\Omega$ :

$$
\begin{aligned}
u(x)= & \int_{\Sigma}\left\{\left[\partial_{n(y)} \Gamma(x-y, k)\right]\left([u(y)]^{+}-[u(y)]^{-}\right)\right. \\
& \left.-\Gamma(x-y, k)\left(\left[\partial_{n} u(y)\right]^{+}-\left[\partial_{n} u(y)\right]^{-}\right)\right\} d S
\end{aligned}
$$




$$
=W\left([u]_{\Sigma}^{+}-[u]_{\Sigma}^{-}\right)(x)-V\left(\left[\partial_{n} u\right]_{\Sigma}^{+}-\left[\partial_{n} u\right]_{\Sigma}^{-}\right)(x), \quad x \in \Omega .
$$

Thus, this formula holds for a solution $u \in H^{1}(\Omega)$ of the Helmholtz equation in the case of a complex wave number $k$ as well as for a radiating solution $u \in H_{l o c}^{1}(\Omega) \cap \operatorname{Som}(\Omega)$ in the case of a real positive wave number $k$.

Note that $[u]_{S}^{ \pm} \in H^{\frac{1}{2}}(S)$ and $\left[\partial_{n} u\right]_{S}^{ \pm} \in H^{-\frac{1}{2}}(S)$ with $S=\partial \Omega_{ \pm}$, and, due to equalities (3.5), we have the inclusions

$$
[u]_{S}^{+}-[u]_{S}^{-} \in \widetilde{H}^{\frac{1}{2}}(\Sigma), \quad\left[\partial_{n} u\right]_{S}^{+}-\left[\partial_{n} u\right]_{S}^{-} \in \widetilde{H}^{-\frac{1}{2}}(\Sigma)
$$

Let us remark here that we have the continuous embedding $H^{\frac{1}{2}}(\Sigma) \hookrightarrow$ $r_{\Sigma} \widetilde{H}^{-\frac{1}{2}}(\Sigma)$ which, along with $(3.7)$, shows that the inclusions in (1.4) are actually necessary for the problem $(P)_{\Sigma}$ to be solvable.

We recall now some properties of the above-introduced potentials and of the corresponding boundary integral operators needed in our further analysis.

We start with the so-called jump relations

$$
\begin{aligned}
& {[V(\psi)]_{S}^{+}=[V(\psi)]_{S}^{-}=: \mathcal{H} \psi, \quad\left[\partial_{n} V(\psi)\right]_{S}^{ \pm}=:\left[\mp 2^{-1} I+\mathcal{K}^{\top}\right] \psi,} \\
& {[W(\varphi)]_{S}^{ \pm}=:\left[ \pm 2^{-1} I+\mathcal{K}\right] \varphi, \quad\left[\partial_{n} W(\varphi)\right]_{S}^{+}=\left[\partial_{n} W(\varphi)\right]_{S}^{-}=: \mathcal{L} \varphi}
\end{aligned}
$$

where $I$ stands for the unit operator, and

$$
\begin{aligned}
& (\mathcal{H} \psi)(x):=\int_{\Sigma} \Gamma(x-y, k) \psi(y) d S, \quad x \in \Sigma, \\
& \left(\mathcal{K}^{\top} \psi\right)(x):=\int_{\Sigma}\left[\partial_{n(x)} \Gamma(x-y, k)\right] \psi(y) d S, \quad x \in \Sigma, \\
& (\mathcal{K} \varphi)(x):=\int_{\Sigma}\left[\partial_{n(y)} \Gamma(y-x, k)\right] \varphi(y) d S, \quad x \in \Sigma, \\
& (\mathcal{L} \varphi)(x):=\lim _{\Omega^{ \pm} \ni z \rightarrow x \in \Sigma} \sum_{j=1}^{2} n_{j}(x) \partial_{z_{j}} W(\varphi)(z), \quad x \in \Sigma .
\end{aligned}
$$

Clearly, $\mathcal{K}$ and $\mathcal{K}^{\top}$ are mutually transposed operators.

The above-introduced integral operators, originally defined for smooth functions with a compact support in $\Sigma$, can be continuously extended to the bounded operators

$$
\begin{array}{lll}
r_{\Sigma} \mathcal{H} \quad: \quad \tilde{H}^{s}(\Sigma) \rightarrow H^{s+1}(\Sigma), & r_{\Sigma} \mathcal{K}: \tilde{H}^{s}(\Sigma) \rightarrow H^{s}(\Sigma), \\
r_{\Sigma} \mathcal{K}^{\top}: \quad \tilde{H}^{s}(\Sigma) \rightarrow H^{s}(\Sigma), & r_{\Sigma} \mathcal{L}: \widetilde{H}^{s+1}(\Sigma) \rightarrow H^{s}(\Sigma),
\end{array}
$$

for arbitrary $s \in \mathbb{R}$. Moreover, they are pseudodifferential operators of order $-1,0,0$, and 1 , respectively.

We also have the following mapping properties of a single- and a double-layer potential,

$$
\begin{aligned}
& V \quad: \quad \tilde{H}^{s}(\Sigma) \rightarrow H^{s+1+\frac{1}{2}}\left(\Omega^{ \pm}\right), \\
& W \quad: \quad \widetilde{H}^{s}(\Sigma) \rightarrow H^{s+\frac{1}{2}}\left(\Omega^{ \pm}\right),
\end{aligned}
$$


for a complex $k$, and

$$
\begin{aligned}
& V \quad: \widetilde{H}^{s}(\Sigma) \rightarrow H_{l o c}^{s+1+\frac{1}{2}}\left(\Omega^{-}\right) \cap \operatorname{Som}\left(\Omega^{-}\right), \\
&: \widetilde{H}^{s}(\Sigma) \rightarrow H^{s+1+\frac{1}{2}}\left(\Omega^{+}\right) \\
& W \quad: \quad \widetilde{H}^{s}(\Sigma) \rightarrow H_{l o c}^{s+\frac{1}{2}}\left(\Omega^{-}\right) \cap \operatorname{Som}\left(\Omega^{-}\right) \\
& \quad: \quad \widetilde{H}^{s}(\Sigma) \rightarrow H^{s+\frac{1}{2}}\left(\Omega^{+}\right)
\end{aligned}
$$

for a real positive $k$.

The proofs of the above assertions can be found, e.g., in [5, 10, 11, 12, 13, 14], where the Fredholm properties of these operators are studied as well.

We apply these results to show the existence and regularity of solutions to our original problem $(P)_{\Sigma}$.

\section{Existence And Regularity Results}

We look for a solution of the problem $(P)_{\Sigma}$ in the form

$$
u(x)=W(\varphi)(x)-V(\psi)(x), \quad x \in \Omega,
$$

where the unknown densities $\varphi$ and $\psi$ are related to the boundary values of the source $u$ and its normal derivative $\partial_{n} u$ by the equations

$$
\varphi=[u]_{S}^{+}-[u]_{S}^{-}, \quad \psi=\left[\partial_{n} u\right]_{S}^{+}-\left[\partial_{n} u\right]_{S}^{-}
$$

due to the general integral representation formula (3.6). We assume that

$$
\varphi \in \widetilde{H}^{\frac{1}{2}}(\Sigma), \quad \psi \in \widetilde{H}^{-\frac{1}{2}}(\Sigma),
$$

in accordance with the inclusions (3.7). Note that the embedding $u \in H^{1}(\Omega)$ for a complex $k$ and the embedding $u \in H_{l o c}^{1}(\Omega) \cap \operatorname{Som}(\Omega)$ for a real positive $k$ are then automatically satisfied.

With the help of jump relations (3.8) and the boundary conditions (1.2)-(1.3) we get the following equations for the densities $\varphi$ and $\psi$,

$$
\begin{aligned}
& r_{\Sigma} \varphi=h_{0} \text { on } \Sigma, \\
& r_{\Sigma} \psi+q r_{\Sigma}\left[2^{-1} \varphi+\mathcal{K} \varphi-\mathcal{H} \psi\right]=h_{1} \text { on } \Sigma .
\end{aligned}
$$

Let us construct the matrix operator

$$
\mathcal{P}:=\left[\begin{array}{cc}
r_{\Sigma} I & 0 \\
q r_{\Sigma}\left[2^{-1} I+\mathcal{K}\right] & r_{\Sigma}[I-q \mathcal{H}]
\end{array}\right]
$$

and consider the vectors

$$
\Phi:=(\varphi, \psi)^{\top}, \quad F:=\left(h_{0}, h_{1}\right)^{\top} .
$$

The simultaneous equations (4.4) and (4.5) can now be written as

$$
\mathcal{P} \Phi=F \text { on } \Sigma
$$

where

$$
\Phi \in \widetilde{H}^{\frac{1}{2}}(\Sigma) \times \widetilde{H}^{-\frac{1}{2}}(\Sigma), \quad F \in r_{\Sigma}\left[\widetilde{H}^{\frac{1}{2}}(\Sigma) \times \widetilde{H}^{-\frac{1}{2}}(\Sigma)\right]
$$


Lemma 4.1. The operator

$$
\mathcal{P}: \widetilde{H}^{\frac{1}{2}}(\Sigma) \times \widetilde{H}^{-\frac{1}{2}}(\Sigma) \rightarrow r_{\Sigma}\left[\widetilde{H}^{\frac{1}{2}}(\Sigma) \times \widetilde{H}^{-\frac{1}{2}}(\Sigma)\right]
$$

is invertible.

Proof. Due to the compactness of $\bar{\Sigma}$, the operator

$$
r_{\Sigma} \mathcal{H}: \widetilde{H}^{-\frac{1}{2}}(\Sigma) \rightarrow r_{\Sigma} \widetilde{H}^{-\frac{1}{2}}(\Sigma)
$$

is compact in view of the mapping property (3.13). Therefore, (4.9) is a compact perturbation of the triangular operator with the unit $\left(r_{\Sigma} I\right)$ invertible operators on the principal diagonal. Consequently, (4.9) is a Fredholm operator with zero index. The homogeneous equation $\mathcal{P} \Phi=0$ implies $\Phi=0$ on $\Sigma$ due to the uniqueness theorem for the problem $(P)_{\Sigma}$. Thus the null space of the operator (4.9) is trivial, which completes the proof.

From this lemma it follows that the nonhomogeneous equation (4.8) is uniquely solvable for arbitrary $F \in r_{\Sigma}\left[\widetilde{H}^{\frac{1}{2}}(\Sigma) \times \widetilde{H}^{-\frac{1}{2}}(\Sigma)\right]$. This immediately leads to the following existence result.

Theorem 4.2. The boundary value problem $(P)_{\Sigma}$ is uniquely solvable for arbitrary data $h_{0}$ and $h_{1}$ satisfying the inclusions (1.4). The solution $u$ is representable in the form (4.1), where

$$
r_{\Sigma} \varphi=h_{0}
$$

and $\psi$ solves the integral equation

$$
r_{\Sigma}[\psi-q \mathcal{H} \psi]=h_{1}-q r_{\Sigma}\left[2^{-1} \varphi+\mathcal{K} \varphi\right] \text { on } \Sigma \text {. }
$$

Moreover, the solution $u$ belongs to the space $H^{1}(\Omega)$ for a complex $k$, and to the space of radiating functions $H_{\text {loc }}^{1}(\Omega) \cap \operatorname{Som}(\Omega)$ for a real positive $k$.

Now we require that the boundary data $h_{0}$ and $h_{1}$ be smoother and derive higher regularity results for the solution pair $(\varphi, \psi)$ of the system $(4.4)-(4.5)$, and for the corresponding solution $u$ of BVP $(P)_{\Sigma}$. All these requirements are assembled in the following assertion.

Theorem 4.3. If the boundary data satisfy the conditions

$$
\left(h_{0}, h_{1}\right) \in r_{\Sigma}\left[\widetilde{H}^{1+s}(\Sigma) \times \widetilde{H}^{s}(\Sigma)\right], \quad-1 / 2<s<1 / 2,
$$

then the solution $u$ to the problem $(P)_{\Sigma}$ possesses the regularity

$$
u \in H^{1+\frac{1}{2}+s}\left(\Omega^{ \pm}\right)
$$

for a complex $k$ and

$$
u \in H_{l o c}^{1+\frac{1}{2}+s}\left(\Omega^{ \pm}\right) \cap \operatorname{Som}(\Omega)
$$

for a real positive $k$. 
Proof. Due to Lemma 4.1 it is evident that the system (4.4)-(4.5) with $h_{0}$ and $h_{1}$ as in $(4.12)$ is solvable and $(\varphi, \psi) \in\left(\widetilde{H}^{\frac{1}{2}}(\Sigma) \times \widetilde{H}^{-\frac{1}{2}}(\Sigma)\right)$. To show a higher regularity we proceed as follows.

First we remark that if $h_{0} \in r_{\Sigma} \widetilde{H}^{1+s}(\Sigma)$, then from (4.10) we have $r_{\Sigma} \varphi=h_{0}$, i.e.,

$$
\varphi \in \widetilde{H}^{1+s}(\Sigma), \quad-1 / 2<s<1 / 2 .
$$

In accordance with the mapping property (3.13), it follows that

$$
r_{\Sigma} \mathcal{K} \varphi \in H^{1+s}(\Sigma) \subset H^{s}(\Sigma)=r_{\Sigma} \widetilde{H}^{s}(\Sigma),
$$

since $-1 / 2<s<1 / 2$ ([15], Ch.1, §11; [16], Ch.4, §4.3).

On the other hand, if $h_{1} \in r_{\Sigma} \widetilde{H}^{s}(\Sigma)$, then from (4.11) we have

$$
r_{\Sigma} \psi-q r_{\Sigma}[\mathcal{H} \psi]=f \text { on } \Sigma
$$

with

$$
f:=h_{1}-q r_{\Sigma}\left[2^{-1} \varphi+\mathcal{K} \varphi\right] \in r_{\Sigma} \widetilde{H}^{s}(\Sigma) \subset r_{\Sigma} \widetilde{H}^{-\frac{1}{2}}(\Sigma) .
$$

Therefore $\psi \in \widetilde{H}^{-\frac{1}{2}}(\Sigma)$ implies

$$
r_{\Sigma} \mathcal{H} \psi \in H^{\frac{1}{2}}(\Sigma) \subset H^{s}(\Sigma)=r_{\Sigma} \widetilde{H}^{s}(\Sigma)
$$

due to the mapping property (3.13) and since $-1 / 2<s<1 / 2$. Then from (4.17) it follows that

$$
\psi \in \widetilde{H}^{s}(\Sigma) .
$$

Now, from the representation formula (4.1) and the inclusions (4.15)-(4.18) we conclude by (3.14) and (3.15) that the solution $u$ to the problem $(P)_{\Sigma}$ has the regularity properties (4.13) and (4.14).

Remark 4.4. By using the Sobolev-Kondrashov embedding theorems (for the two-dimensional case $\Omega \subset \mathbb{R}^{2}$ ), from (4.13) and (4.14) we conclude that $u \in$ $C^{\frac{1}{2}+s}\left(\overline{\Omega^{ \pm}}\right)$with $0<s<1 / 2$ (see, e.g., [16], Ch. 2, §2.8.1). Moreover, $\left[\partial_{n} u\right]_{S}^{ \pm} \in$ $H^{s}(S)$ exist in the usual trace sense.

Remark 4.5. We note here that in the three-dimensional case we have to construct the layer potentials with the help of the fundamental function

$$
\Gamma(x, k):=-\frac{1}{4 \pi} \frac{\exp \{i k|x|\}}{|x|}
$$

which satisfies the Sommerfeld radiation condition (1.6). The jump relations and mapping properties of these layer potentials and the corresponding boundary integral operators are absolutely the same as described in Section 3. Therefore the main existence and regularity results obtained in Section 4, Lemma 4.1 and Theorems 4.2 and 4.3, remain valid in the three-dimensional case as well. The Hölder continuity result for the solution $u$ now reads as $u \in C^{s}\left(\overline{\Omega^{ \pm}}\right)$, with $0<s<1 / 2$, in accordance with the Sobolev-Kondrashov embedding theorems for the three-dimensional case $\Omega \subset \mathbb{R}^{3}$. However, we still have the embedding $\left[\partial_{n} u\right]_{S}^{ \pm} \in H^{s}(S)$ which shows that the generalized traces $\left[\partial_{n} u\right]_{\Sigma}^{ \pm}$actually exist in the usual trace sense. 


\section{REFERENCES}

1. I. J. LaHAiE, Function theoretic techniques for the electromagnetic scattering by a resistive wedge. Radiation Laboratory Techn. Report: Vol. 2. Univ. Michigan, Ann Arbor, 1981.

2. E. Meister and F.-O. Speck, Modern Wiener-Hopf methods in diffraction theory. Ordinary and partial differential equations, Vol. II (Dundee, 1988), 130-171, Pitman Res. Notes Math. Ser., 216, Longman Sci. Tech., Harlow, 1989.

3. A. BÜyükaksoy, F. Birbir, E. ErdoĞan, and G. UzGören, Plane wave diffraction by a rectangular groove in a reactive surface. Direct and inverse electromagnetic scattering (Gebze, 1995), 101-120, Pitman Res. Notes Math. Ser., 361, Longman, Harlow, 1996.

4. R. T. Seeley, Singular integrals and boundary value problems. Amer. J. Math. 88(1966), 781-809.

5. M. Costabel and W. L. Wendland, Strong ellipticity of boundary integral operators. J. Reine Angew. Math. 372(1986), 34-63.

6. W. McLean, Strongly elliptic systems and boundary integral equations. Cambridge University Press, Cambridge, 2000.

7. I. N. Vekua, On metaharmonic functions. (Russian) Trudy Tbiliss. Mat. Inst. Razmadze 12(1943), 105-174.

8. D. Colton and R. Kress, Inverse acoustic and electromagnetic scattering theory. Second edition. Applied Mathematical Sciences, 93. Springer-Verlag, Berlin, 1998.

9. L. P. Castro and D. Natroshvili, The reactance wave diffraction problem by a strip in a scale of Bessel potential spaces. To appear.

10. G. I. Eskin, Boundary value problems for elliptic pseudodifferential equations. Translated from the Russian by S. Smith. Translations of Mathematical Monographs, 52. American Mathematical Society, Providence, R.I., 1981.

11. R. Duduchava, On multidimensional singular integral operators. I, II. J. Operator Theory 11(1984), No. 1, 41-76, 199-214.

12. R. Duduchava, D. Natroshvili, and E. Shargorodsky, Boundary value problems of the mathematical theory of cracks. Tbiliss. Gos. Univ. Inst. Prikl. Mat. Trudy 39(1990), $68-84$.

13. E. Shargorodsky, An $L_{p}$-analogue of the Vishik-Eskin theory. Mem. Differential Equations Math. Phys. 2(1994), 41-146.

14. R. Duduchava, D. Natroshvili, and E. Shargorodsky, Basic boundary value problems of thermoelasticity for anisotropic bodies with cuts. II. Georgian Math. J. 2(1995), No. 3, 259-276.

15. J.-L. Lions and E. Magenes, Problèmes aux limites non homognes et applications. Vol. 1. Travaux et Recherches Mathématiques, No. 17 Dunod, Paris, 1968.

16. H. TRIEBEL, Interpolation theory, function spaces, differential operators. VEB Deutscher Verlag der Wissenschaften, Berlin, 1978.

(Received 2.11.2005)

\section{Authors' Addresses:}
L. P. Castro
D. Natroshvili
Department of Mathematics \& Research
Department of Mathematics
Unit "Matemática e Aplicações"
Georgian Technical University
University of Aveiro, 3810-193 Aveiro,
Portugal
77, M.Kostava St., Tbilisi 0175
E-mail: lcastro@mat.ua.pt
Georgia
E-mail:natrosh@hotmail.com 